\title{
Superficial siderosis of the central nervous system many years after neurosurgical procedures
}

\author{
M O McCarron, P A Flynn, C Owens, I Wallace, M Mirakhur, J M Gibson, V H Patterson
}

J Neurol Neurosurg Psychiatry 2003;74:1326-1328

Recurrent haemorrhage into the subarachnoid space causes superficial siderosis, which clinically manifests as cerebellar ataxia, sensorineural hearing loss, and myelopathy. Two patients developed clinical, radiological, and biochemical evidence of superficial siderosis many years after surgery. One had two posterior fossa operations, a left temporal craniectomy, and radiotherapy for a presumed brain tumour before developing clinical evidence of superficial sidersosis 37 years later. The other had small bilateral subdural collections from recurrent shunt revisions following posterior fossa surgery for a Chiari malformation, and then developed deafness and ataxia. The first patient currently has the longest recorded delay between presumed subarachnoid bleeding and clinical manifestations of superficial siderosis. Both patients provide further evidence that superficial siderosis of the central nervous system, a progressive neurodegenerative vascular condition, may be a delayed complication of neurosurgical procedures.

$\mathrm{R}$ ecurrent or persistent haemorrhage into the subarachnoid space causes superficial siderosis of the central nervous system (CNS). This rare condition results in the triad of cerebellar ataxia, sensorineural hearing loss, and a myelopathy. ${ }^{1}$ The diagnosis has only been made during life since 1965. An abundance of microglia and Bergmann glia in the molecular layer of the cerebellum, and susceptible glial ensheathment of the eighth cranial nerve are thought to account for the prominence of ataxia and deafness in superficial siderosis. ${ }^{2}$ The usual sources of the subarachnoid bleeding are dural abnormalities, vascular lesions, and tumours. ${ }^{3-6}$ Nowadays the diagnosis can be confirmed with magnetic resonance imaging (MRI) of brain, which provides evidence of haemosiderin in the form of a rim of hypointensity around the brain stem on T2 weighted images. ${ }^{1}$

We report two patients who each had had several neurosurgical procedures and in whom the delay in symptom onset followed by a slowly progressive course eventually led to the diagnosis of superficial siderosis of the CNS.

\section{CASE REPORTS}

\section{Case 1}

An eight year old boy presented in June 1962 with a four month history of right sided incoordination. An exploratory operation of his posterior fossa revealed no cause except a suspected anomalous blood vessel. This was ligated at a second operation. There was some initial improvement before subsequent deterioration occurred. Headache, daily vomiting, increasing right hemiparesis, dysphasia, and a right homonymous hemianopia developed. Emergency temporal craniectomy was undertaken in November 1962. No definite pathological tumour tissue was identified. It was assumed that the patient had a left temporal tumour, possibly a cystic astrocytoma, and he was treated with radiotherapy. He was discharged home, where he gradually improved. He had some learning difficulties but gained independence, living alone and working in a factory, despite a mild right hemiparesis and ataxia.

In 1999, 37 years after the neurosurgery, he gradually became more ataxic, particularly in the trunk, with falls at work and at home. He had also developed deafness and eventually could no longer work.

A T2 weighted MRI scan showed hypointense signal around the brain stem and in the folia of the cerebellum consistent with haemosiderin deposition (fig lA). An old left temporal craniectomy defect and postoperative temporal encephalomalacia were also noted. CSF protein and ferritin were raised and spectrophotometry revealed oxyhaemoglobin and bilirubin (table 1). Audiometry confirmed high frequency deafness, and brain stem evoked potentials were unrecordable at $90 \mathrm{~dB}$. All the findings were consistent with superficial siderosis of the CNS

\section{Case 2}

A 22 year old man presented in 1979 with a two year history of headaches, gait unsteadiness, and dizziness on neck movement. Examination revealed lateral gaze evoked nystagmus and an ataxic gait. A posterior fossa craniectomy showed tonsillar herniation to the level of $\mathrm{Cl} / \mathrm{C} 2$. Postoperative headache, nausea, and vomiting occurred. A metrizimide myelogram showed complete obstruction at $\mathrm{C} 2$, and at re-exploration of the posterior cranial fossa the spinous processes and laminae were removed from C2 and C3. Within six months neck stiffness, headaches, and vomiting occurred. Computed tomography of the brain showed enlarged ventricles. A ventriculoatrial shunt was inserted. However, headaches, photophobia, and neck pain remained prominent symptoms. In 1982 the shunt was converted to ventriculo-peritoneal. Intracranial pressure monitoring revealed no increased pressure, but rather postural falls in pressure. In 1981, 1982, 1983, and 1986 the CSF had a xanthrochromic appearance, and there was a peak on spectrophotometry consistent with bilirubin. In 1982, four vessel angiography was normal.

The patient gradually deteriorated with headaches, poor balance (necessitating a wheelchair), tinnitus, high frequency hearing loss, oscillopsia, and detrusor instability. Numerous shunt revisions were of little help and brain MRI on two occasions in the last 10 years showed T2 weighted low signal changes around the posterior fossa structures and spinal cord, consistent with haemosiderin deposition. Chronic subdural collections were also present on brain MRI in 2001 (fig 1B). In August 2002 a repeat CSF analysis from a shunt reservoir confirmed raised ferritin, although the net bilirubin absorbance was below the reference range for evidence of subarachnoid haemorrhage (table 1). Brain stem evoked potentials were unrecordable. 

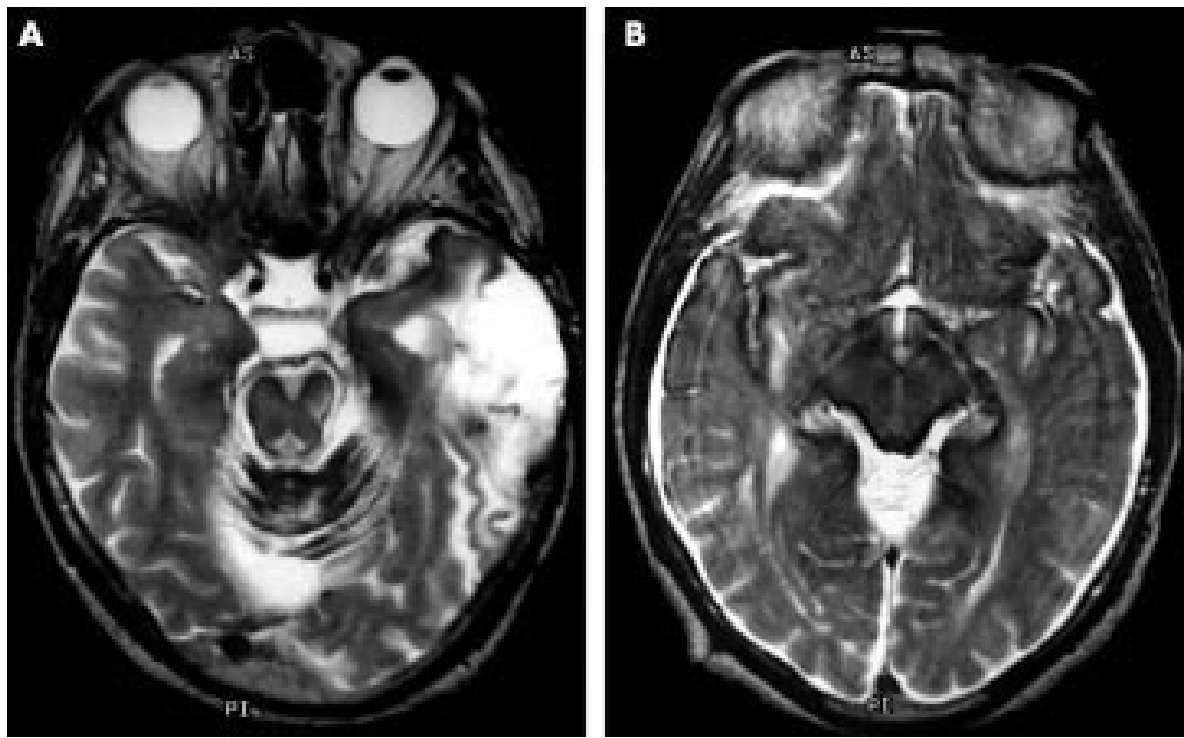

Figure 1 (A) Turbo-spin T2 axial magnetic resonance scan of brain from patient 1 showing low signal around the brain stem and cerebellum because of haemosiderin deposition. There is also an old left temporal craniectomy defect and postoperative encephalomalacia. (B) Turbo-spin T2 axial magnetic resonance brain scan from patient 2 showing low signal from the brain stem surface, consistent with haemosiderin deposition. Bilateral subdural collections are also present.

\begin{tabular}{|c|c|c|c|}
\hline & & Patient 1 & Patient 2 \\
\hline CSF & $\begin{array}{l}\text { White cell count } \\
\text { Red cell count } \\
\text { Protein }(\mathrm{g} / \mathrm{l}) \\
\text { Glucose }(\mathrm{mmol} / \mathrm{l}) \\
\text { Ferritin }(<12 \mu \mathrm{g} / \mathrm{ll}) \\
\text { Spectrophotometry } \\
\text { Net bilirubin absorbance* }(<0.007)\end{array}$ & $\begin{array}{l}\text { December } 2001 \\
<1 \\
40 \\
1.2 \\
3.8 \\
90 \\
\text { Oxyhaemoglobin } \\
0.018\end{array}$ & $\begin{array}{l}\text { August } 2002 \\
6 \\
2355 \\
1.09 \\
3.7 \\
31 \\
\text { No oxyhaemoglobin } \\
0.003\end{array}$ \\
\hline \multicolumn{2}{|c|}{$\begin{array}{l}\text { Blood glucose }(\mathrm{mmol} / \mathrm{l}) \\
\text { BAEP } \\
\text { VEP }\end{array}$} & $\begin{array}{l}4.2 \\
\text { Absent at } 90 \mathrm{~dB} \\
\text { Normal latency }\end{array}$ & $\begin{array}{l}4.4 \\
\text { Absent at } 90 \mathrm{~dB} \\
\text { Normal latency }\end{array}$ \\
\hline
\end{tabular}

\section{DISCUSSION}

Recurrent or persistent subarachnoid bleeding, which leads to superficial siderosis of the CNS, results from deposition of haemosiderin in the cerebrum, cerebellum, spinal cord, and cranial nerves. Although the pathogenesis is not fully understood, haem is released, which, through haem oxygenase and ferritin, then results in haemosiderin deposition. Two types of glial cell seem to play key roles: microglia synthesise ferritin, and Bergmann glia are the source of iron and haem responsive ferritin repressor protein. ${ }^{27}$ The triad of clinical features of ataxia, deafness, and pyramidal signs can be accompanied by nystagmus, anosmia, pupillary anisocoria, or sluggish/absent light reflexes and dementia. ${ }^{18}$ Although the spinal cord often contains haemosiderin, clinical signs of anterior horn cell dysfunction are unusual. ${ }^{9}$

Nearly half the patients with superficial siderosis have no identifiable cause for chronic or recurrent subarachnoid haemorrhage. Bleeding sources from dural pathology-for example hemispherectomy or cervical root avulsion, tumours such as ependymoma or cerebellar astrocytomas, ${ }^{4}{ }^{6}$ and vascular abnormalities ${ }^{10}$ - have all been documented. An increasing number of non-surgical causes have, however, been implicated, ${ }^{1}$ including transthyretin amyloidosis. ${ }^{11}$ It is tempt- ing to speculate that silent cortical haemorrhage in sporadic cerebral amyloid angiopathy (CAA) caused by amyloid $\beta$ protein may also explain a proportion of the idiopathic cases, but primary subarachnoid haemorrhage is very unusual in patients with $\mathrm{CAA}^{12}$ In addition, many of the idiopathic patients with superficial siderosis are less than 60 years of age, when sporadic CAA is less likely. ${ }^{13}$

The role of radiotherapy in the aetiology of superficial siderosis remains unclear. Patient 1 received radiotherapy. A systematic search for superficial siderosis in 21 patients who had cerebellar tumours found no evidence of superficial siderosis at a median of 14 years follow up. ${ }^{4}$ Other reports support the hypothesis that posterior fossa surgery, ${ }^{3-6}$ not just for tumours, ${ }^{14}$ may contribute to recurrent subarachnoid bleeding. The chronic subdural collections in patient 2, who had a Chiari malformation, are also a possible aetiology; one previous report documented superficial siderosis as an apparent incidental finding in a patient with chronic subdural membranes. ${ }^{15}$ However, the vulnerability of the cerebellum may explain the apparent excess of superficial siderosis in patients who have had posterior fossa surgery, a feature of both our patients. It has been hypothesised that surgery may provide access to the CSF for a bleeding source..$^{14}$ 
In patient 1 there was a 37 year delay between symptom onset and the neurosurgical interventions combined with radiotherapy. This represents the longest presymptomatic period documented, to our knowledge, between the presumed onset of subarachnoid bleeding and clinical manifestations of superficial siderosis. ${ }^{15}$ As found in one of our patients, previous cases have also shown variation in the detection of CSF bilirubin over time, probably reflecting episodic subarachnoid bleeding. ${ }^{1}$ Raised CSF protein, iron, and ferritin, as well as increased numbers of CSF siderophages, have been documented in some but not all patients with superficial siderosis. Importantly for potential therapeutic interventions, changes on MRI predate clinical features, in that asymptomatic patients with superficial siderosis have been identified.

Unfortunately treatment is of uncertain value. Experimental evidence of inhibition of haem oxygenase (the source of the excess tissue iron) is a promising development. ${ }^{16}$ Surgical correction of recognised bleeding sources is recommended, but the evidence remains anecdotal. ${ }^{17}{ }^{18}$ Iron chelation with desferrioxamine (deferoxamine) or trientine ${ }^{19}$ is recommended, but again there is a very limited evidence and no randomised controlled studies. ${ }^{1}$

The two patients in this report highlight the need to consider superficial siderosis as a possible very late neurosurgical complication, particularly in patients with slowly progressive ataxia and deafness. Further knowledge of potential iatrogenic causes (surgery or radiotherapy), pathophysiology, and prevention is required in this underrecognised neurodegenerative vascular condition.

\section{Authors' affiliations}

M O McCarron, C Owens, J M Gibson, V H Patterson, Department of Neurology, Royal Victoria Hospital, Belfast, UK

P A Flynn, Department of Neuroradiology, Royal Victoria Hospital

I Wallace, M Mirakhur, Department of Neuropathology, Royal Victoria Hospital

Competing interests: none declared

Correspondence to: Dr Mark McCarron, Department of Neurology, Royal Victoria Hospital, Belfast BT12 6BA, UK; markmccarron@doctors.org.uk

Received 13 February 2003

Accepted 7 April 2003

\section{REFERENCES}

1 Fearnley JM, Stevens JM, Rudge P. Superficial siderosis of the central nervous system. Brain 1995;118:1051-66.

2 Koeppen AH, Dickson AC, Chu RC, et al. The pathogenesis of superficial siderosis of the central nervous system. Ann Neurol 1993;34:646-53

3 Janss AJ, Galetta SL, Freese A, et al. Superficial siderosis of the central nervous system: magnetic resonance imaging and pathological correlation. Case report. J Neurosurg 1993;79:756-60.

4 Anderson NE, Sheffield S, Hope JK. Superficial siderosis of the central nervous system: a late complication of cerebellar tumors. Neurology 1999;52:163-9.

5 Kassubek J, Obert M, Lucking $\mathrm{CH}$. Superficial siderosis of the CNS: a late complication of cerebellar tumors. Neurology 1999;53:1893-4.

6 McCarron MO, McKinstry CS, Gibson JM. Superficial siderosis 20 years after brain tumour. Lancet Neurol 2002;1:326-6.

7 Koeppen AH, Dentinger MP. Brain hemosiderin and superficial siderosis of the central nervous system. J Neuropathol Exp Neurol 1988:47:249-70.

8 Pelak VS, Galetta SL, Grossman RI, et al. Evidence for preganglionic pupillary involvement in superficial siderosis. Neurology 2002:53:1130-2.

9 Turner B, Wills AJ. Superficial siderosis with anterior horn cell dysfunction. J Neurol Neurosurg Psychiatry 2002;72:274-80.

10 Li KW, Haroun RI, Clatterbuck RE, et al. Superficial siderosis associated with multiple cavernous malformations: report of three cases. Neurosurgery 2001:48:1147-50.

11 Mascalchi M, Salvi F, Pirini MG, et al. Transthyretin amyloidosis and superficial siderosis of the CNS. Neurology 1999;53:1498-503.

12 Yamada $M$, Itoh $Y$, Otomo $E$, et al. Subarachnoid haemorrhage in the elderly: a necropsy study of the association with cerebral amyloid angiopathy. J Neurol Neurosurg Psychiatry 1993;56:543-7.

13 Greenberg SM, Rebeck GW, Vonsattel JP, et al. Apolipoprotein $E \in 4$ and cerebral hemorrhage associated with amyloid angiopathy. Ann Neurol 1995;38:254-9

14 Kumar A, Aggarwal S, Willinsky R, et al. Posterior fossa surgery: an unusual cause of superficial siderosis. Neurosurgery 1993;32:455-7.

15 Parnes SM, Weaver SA. Superficial siderosis of the central nervous system: a neglected cause of sensorineural hearing loss. Otolaryngol Head Neck Surg 1992;107:69-77

16 Koeppen AH, Dickson AC. Tin-protoporphyrin prevents experimental superficial siderosis in rabbits. J Neuropathol Exp Neurol 2002;61:689-701.

17 Haroun RI, Li KW, Rigamonti D. Surgical resection of a cerebral arteriovenous malformation for treatment of superficial siderosis: case report. Surg Neurol 2000;53:554-8.

18 Schievink WI, Apostolides PJ, Spetzler RF. Surgical treatment of superficial siderosis associated with a spinal arteriovenous malformation. Case report. J Neurosurg 1998;89:1029-31.

19 River Y, Honigman S, Gomori JM, et al. Superficial hemosiderosis of the central nervous system. Mov Disord 1994;9:559-62.

20 Allen K, Beetham R, Fahie-Wilson MN, et al. Proposed national guidelines for analysis of cerebrospinal fluid for bilirubin in suspected subarachnoid haemorrhage. United Kingdom National External Quality Assessments Service (UKNEQAS), 2001. 